\title{
RETAINING HIGH AREAL IN-PLANE MAGNETIC ENERGY DENSITY OVER LARGE MAGNETIC THICKNESS: A PERMANENT MAGNETIC MICROLAMINATION APPROACH BASED ON SEQUENTIAL MULTILAYER ELECTROPLATING

\author{
Yuan $\mathrm{Li}^{i^{*}}$, Jooncheol Kim ${ }^{2}$, Minsoo Kim ${ }^{2}$, Andac Armutlulu ${ }^{2}$ and Mark G. Allen ${ }^{l}$ \\ ${ }^{1}$ University of Pennsylvania, Philadelphia, Pennsylvania, USA \\ ${ }^{2}$ Georgia Institute of Technology, Atlanta, Georgia, USA
}

\begin{abstract}
Many magnetic MEMS devices such as magnetic-based microscale energy harvesters rely on the availability of microscale permanent magnets capable of generating significant magnetic fluxes. Ideally, these magnets would be able to be integrated with MEMS in a batch-fabrication-compatible manner. A number of thin film permanent magnets possessing excellent intrinsic magnetic properties have been discussed in the literature. In order to achieve higher extrinsic properties such as magnetic flux, an intuitive approach is to simply increase the magnetic film thickness. However, it is observed that as the magnetic film thickness increases, the intrinsic magnetic properties (such as remanence and maximum energy product) often deteriorate, limiting the maximum achievable magnetic fluxes from these small-scale integrated magnets. In this work, we present a microfabricated permanent magnet with a multilayer structure that preserves the high magnetic energy density (and resultant magnetic flux density) of thinner magnetic films while simultaneously achieving a significant magnetic thickness and resultant extrinsic properties. The fabrication process relies on sequential multilayer electroplating: alternating layers of relatively thin (in microns) magnetic films and non-magnetic materials were electrodeposited in a multilayer fashion realizing a laminated permanent micromagnet up to a total magnetic thickness of $80 \mu \mathrm{m}$. A maximum energy product as high as $16.2 \mathrm{~kJ} / \mathrm{m}^{3}$ ( 70\% of the value of a $1-\mu \mathrm{m}$-thick thin film) was retained in the laminated permanent micromagnet, and a $30 \%$ improvement over a CoNiP nonlaminated film with the same magnetic thickness has been successfully achieved. This fabrication approach could potentially be adapted to other permanent magnet materials systems.
\end{abstract}

\section{INTRODUCTION}

A key challenge in magnetic MEMS is the realization of large-volume, high-energy-product permanent micromagnets that can be deposited in a fully-integrated and CMOS-compatible manner. Such magnets are essential in applications ranging from biasing of magnetic sensors in a portable compass [1] to high force MEMS magnetic actuators [2] to energy harvesting [3]. As an example, the generation of voltage in a permanent magnet energy harvester depends on the rate of change of flux (rather than flux density) according to Faraday's law of induction [4]. For a vibration-based energy harvester operating at a given frequency, increasing the rate of change of the magnetic flux is achieved by increasing the total flux itself [4]. In an in-plane permanent magnet, magnetic flux can be increased by increasing the thickness of the magnet, as long as this thickness increase does not cause a concomitant decrease in intrinsic magnetic properties such as flux density or energy product. Although a number of thin films with high magnetic energy density have been shown [5-8], typically the energy density falls rapidly as the film thickness increases [2, 8-10], resulting in weakened permanent micromagnets which are of limited utility in the applications described above [11].
To address this issue, we propose laminated permanent micromagnets as illustrated in Figure 1. Since the magnetic properties of thick films degrade as film thickness increases, stacking multiple thin magnetic films with preserved properties can be an effective way to achieve considerable overall magnetic thickness while simultaneously retaining good magnetic properties. To achieve this structure, we exploited sequential multilayer electroplating [12], in which individual layers of relatively thin film magnets were electrodeposited in a multilayer fashion to achieve a laminated permanent magnetic structure in a stacked configuration as shown in Figure 1.

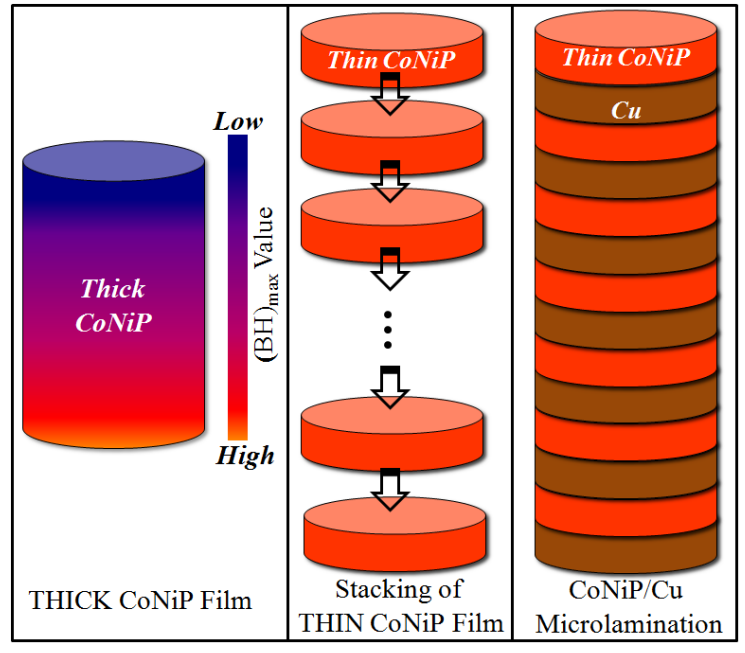

Figure 1: Illustration of the concept of laminated hard magnets (not drawn to scale). (Left) The intrinsic properties of thick electrodeposited magnetic films tend to decrease with thickness. (Center) Conceptually, stacking individual films can produce thick magnets with preserved properties. (Right) Implementation of stacking in-situ using sequential multilayer electrodeposition.

\section{DESIGN AND FABRICATION}

Material Selection

The laminated hard magnetic film is comprised of two types of component layers: a hard magnetic material to act as a functional layer and a nonmagnetic interlamination layer to act to 're-seed' the growth of high-energy-product magnetic films.

The candidate hard magnetic material for this work should be compatible with electrodeposition (due to the relative economy and large deposition rates achievable for films of substantial overall thickness) and should have a controllable direction of magnetic anisotropy. The multilayer structure of Figure 1 has magnetic films with a high aspect ratio of in-plane dimension to thickness, resulting in a high in-plane magnetic shape anisotropy. A magnetic material with controllable magnetocrystalline anisotropy could potentially 
be engineered to align the shape and crystalline anisotropy, thereby boosting overall magnetic performance.

The potential suitable hard magnetic materials include RE (rare earth) magnetic alloys ( $\mathrm{NdFeB}$ and $\mathrm{SmCo}$ ), equiatomic Pt-TM (Transition Metal) alloys (FePt $\mathrm{L1}_{0}$ and $\mathrm{CoPt} \mathrm{L}_{0}$ ), Co-rich hexagonal alloys (CoNiP, CoNiMnP, CoPt, CoPtP) and others [5, 6]. RE magnetic alloys often seen in bulk-scale applications could be integrated in MEMS [13] but with restrictive processing conditions [10]. Equiatomic Pt-TM alloys with attractive performance commonly require either a high temperature deposition environment or relatively high temperature post-process annealing. Among the Co-rich hexagonal alloys, CoNiP can not only be readily electroplated but also be conveniently tuned with bath compositions [14] and electroplating parameters [15] to yield in-plane magnetocrystalline anisotropy with significant in-plane maximum energy product.

In addition to being electrically conductive (to support the next deposition of magnetic material) and electrodepositable, it is hypothesized that the interlamination material should have excellent planarization properties such that the surface of the underlying magnetic material is fully reset for subsequent depositions. $\mathrm{Cu}$ plated from a commercial copper bath (Grobet, Clean Earth $\mathrm{Cu}$-mirror solution) containing brighteners and levelers was chosen as the interlamination material for low surface roughness [16].

\section{Magnetic Film Optimization}

Park et al. [14] showed that the magnetic properties (in-plane/out-of-plane $(\mathrm{BH})_{\max }$ and anisotropy) of CoNiP were strongly influenced by $\mathrm{NaH}_{2} \mathrm{PO}_{2}$ concentrations in the bath. Kirkwood et al. [15] demonstrated a strong correlation between applied current density and c-axis orientation (perpendicular/longitudinal) to the film plane. Based on these results, the plating bath and deposition parameters summarized in Table I were used in this work to achieve significant longitudinal (in-plane) magnetic anisotropy and energy density in the magnetic films. No agitation was applied and $\mathrm{Ni}$ sheet was used as an anode for Ni ion replenishment. Due to the lack of Co ion replenishment as plating continued, in order to ensure compositional uniformity of each magnetic film layer in the multilayer structure, the bath volume was adjusted such that the consumption of Co was less than $1 \%$ of total dissolved ions in the bath for every batch.

Table I: Bath composition for CoNiP thin films.

\begin{tabular}{|cc|}
\hline Chemicals & Concentration $(\boldsymbol{g} / \mathbf{L})$ \\
\hline $\mathrm{NiCl}_{2} \bullet 6 \mathrm{H}_{2} \mathrm{O}$ & 47.5 \\
\hline $\mathrm{CoCl}_{2} \bullet 6 \mathrm{H}_{2} \mathrm{O}$ & 49.0 \\
\hline $\mathrm{NaH}_{2} \mathrm{PO}_{2} \bullet \mathrm{H}_{2} \mathrm{O}$ & 3.0 \\
\hline $\mathrm{NaCl}$ & 40.9 \\
\hline $\mathrm{H}_{3} \mathrm{BO}_{3}$ & 24.7 \\
\hline Saccharin & 0.88 \\
\hline Current Density: $20 \mathrm{~mA} / \mathrm{cm}^{2}, \mathrm{pH}: 2.2$ \\
\hline
\end{tabular}

Figure 2 shows typical in-plane and out-of plane magnetic hysteresis loops, measured by vibrating sample magnetometry, of a single layer 1- $\mu \mathrm{m}$-thick CoNiP thin film deposited using the conditions discussed above. As desired, significant magnetic remanence in the longitudinal direction was observed. EDX results showed the $1 \mu \mathrm{m}$ thin films had a composition (in Atomic \%) of 78 $\mathrm{Co} \%, 13 \% \mathrm{Ni}$ and $9 \% \mathrm{P}$. Both the thin film composition and the magnetic performance are similar to literature values $[14,15]$.

\section{Microlamination Fabrication}

The fabrication sequence is summarized in Figure 3. An insulating layer of silicon dioxide was deposited on a silicon wafer by plasma enhanced chemical vapor deposition (PECVD). An electroplating seed layer structure $(\mathrm{Ti} / \mathrm{Cu} / \mathrm{Ti})$ was then sputter-deposited (Figure 3(a), (b)). A thick photoresist (NR 21-20000P, Futurrex, Inc.) mold was then formed consisting of an array of circles, each $16 \mathrm{~mm}^{2}$ in area (Figure 3(c), (d)). To eliminate possible oxidation of the $\mathrm{Cu}$ seed layer in the sandwich $\mathrm{Ti} / \mathrm{Cu} / \mathrm{Ti}$ seed layer structure, the top layer of $\mathrm{Ti}$ was stripped in dilute hydrofluoric acid just before electroplating commenced (Figure 3(e)). Robotically-assisted sequential multilayer electrodeposition of CoNiP and $\mathrm{Cu}$ layers was then carried out in a dual bath system with the customized CoNiP bath (with plating conditions detailed in Table I) and the commercial $\mathrm{Cu}$ bath (at a current density of 20 $\mathrm{mA} / \mathrm{cm}^{2}$ ). Individual layer thicknesses of both the CoNiP and $\mathrm{Cu}$ layers were set to $1 \mu \mathrm{m}$ by control of the deposition time (Figure 3(f)). After deposition, the photoresist mold was removed and the morphology of resultant micromagnets (Figure $3(\mathrm{~g})$ ) were characterized by a scanning electron microscope (SEM) equipped with energy-dispersive X-ray spectroscopy (EDX). The magnetic properties were characterized by vibrating sample magnetometery (VSM). No correction for demagnetization effects was applied to the data presented below.

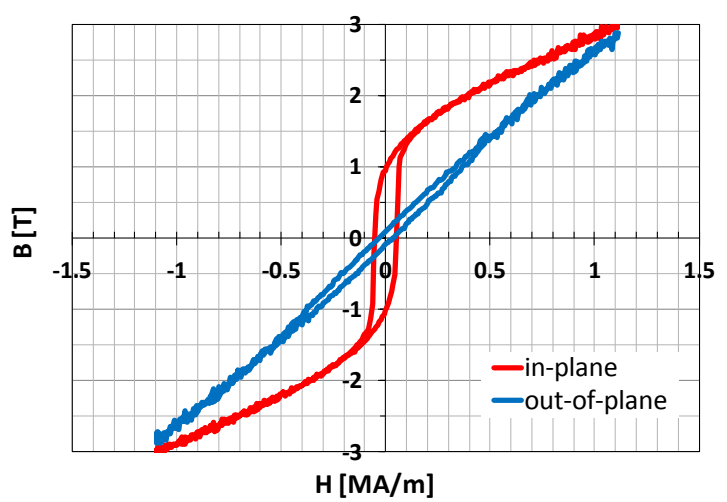

Figure 2: Typical in-plane and out-of-plane hysteresis loops of 1- $\mu$ m-thick CoNiP films.

A cross-sectional SEM image of the fabricated magnetic microlamination is shown in Figure 4(a), comprising a 10-pair $\mathrm{CoNiP}(1 \mu \mathrm{m}) / \mathrm{Cu}(1 \mu \mathrm{m})$ laminated magnet. A short selective $\mathrm{Cu}$ wet etch was performed to create contrast between the layers; the brighter, protruding layer and darker, receding layer are $\mathrm{CoNiP}$ and $\mathrm{Cu}$, respectively (see Figure 4(b)).

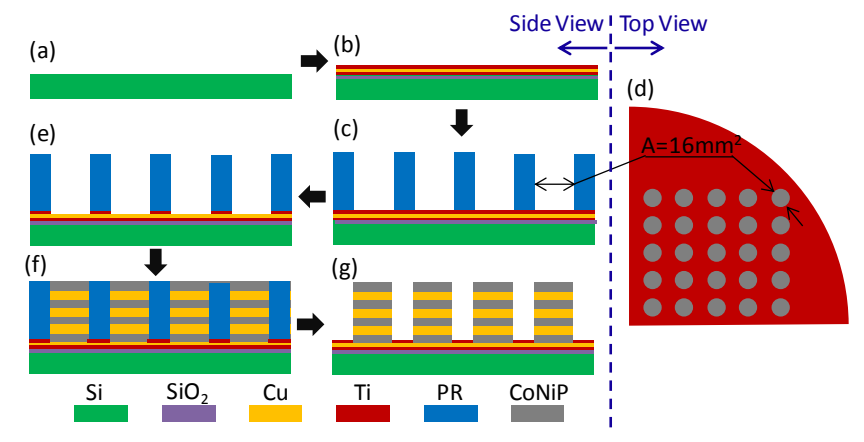

Figure 3: Fabrication process flow. 


\section{RESULTS AND DISCUSSION}

Magnetic Characterization

In a multilayer structure such as the one fabricated here, the relevant thickness for comparing the magnetic properties of various structures is the total magnetic thickness $\left(t_{M}\right)$ : the sum of the thicknesses of the magnetic lamination layers. The magnetic thickness will always be less than or equal to the total thickness. For a single layer magnetic film, the magnetic thickness and the total thickness are equal.

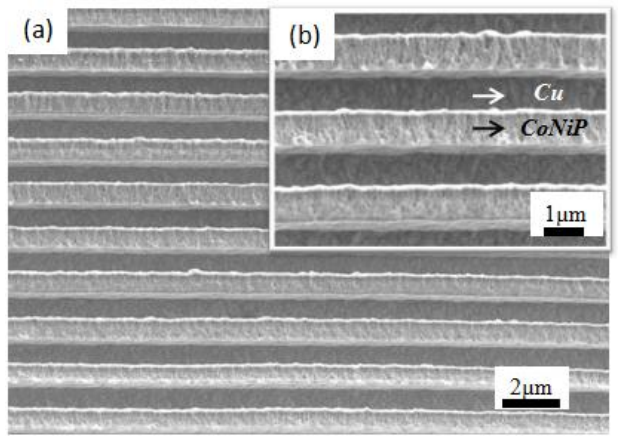

Figure 4: Cross-sectional SEM images of (a) a 10-pair $\mathrm{CoNiP}(1 \mu \mathrm{m}) / \mathrm{Cu}(1 \mu \mathrm{m})$ microlamination and $(b)$ an enlarged view.

Figure 5 compares the dependence of various in-plane magnetic properties of $\mathrm{CoNiP} / \mathrm{Cu}$ multilayer and $\mathrm{CoNiP}$ single layer films measured by VSM as a function of total magnetic thickness $\left(t_{M}\right)$. Figure 5(a) shows the variation of in-plane maximum energy product $\left((\mathrm{BH})_{\max }\right)$ versus $t_{M}$. It is evident that the $(\mathrm{BH})_{\max }$ of the CoNiP was well-maintained in the microlamination configuration up to a total magnetic thickness of $80 \mu \mathrm{m}$, while a single $80 \mu \mathrm{m}$ thick CoNiP film shows substantial degradation of $(\mathrm{BH})_{\max } \cdot(\mathrm{BH})_{\max }$ as high as $16.2 \mathrm{~kJ} / \mathrm{m}^{3}$ was achieved even at a large magnetic thickness of $80 \mu \mathrm{m}$, an approximately $30 \%$ improvement over nonlaminated CoNiP films of the same magnetic thickness.

Figures 5(b) and (c) compare the remanence and coercivity of single layer and multilayer films as a function of magnetic thickness. The deterioration of $(\mathrm{BH})_{\max }$ in single layer thick CoNiP films can be primarily attributed to reduction of remanence rather than coercivity. The decrease in $\mathrm{B}_{\mathrm{r}}$ with increasing film thickness in single layer films may originate from the randomization of crystallographic alignment (well-ordered in the case of thin films) as the film thickness increases [7, 17]. The coercivity of single layer CoNiP films exhibited a slight increase with increased magnetic thickness (a similar trend is also found in the CoNiMnP system [8]).

\section{Figure of Merit}

The utility of the microlamination technique can be further demonstrated by considering maximum areal magnetic energy density (maximum energy per unit area, or the product of $(\mathrm{BH})_{\max }$ and $t_{M}$ ), as an appropriate figure of merit for integrated MEMS magnets. It is a manifestation of how much energy per unit area one can obtain from a microfabricated magnet given a MEMS-constrained footprint. Figure 6 compares the maximum areal energy densities of films with same magnetic thicknesses from this work and the literature [2, 8-10]. Figure 6 (a) shows all data collected for comparison. For better illustration, some data were selected and grouped into thickness ranges (Figure 6 (b)). As can be seen in Figure 6 (b), in the case of MEMS magnets deposited in a CMOS-compatible and fully integrated manner, the microlaminated magnets presented in this work can achieve the highest maximum areal energy density among the materials studied, due to their ability to maintain high $(\mathrm{BH})_{\max }$ at very large total magnetic thicknesses.
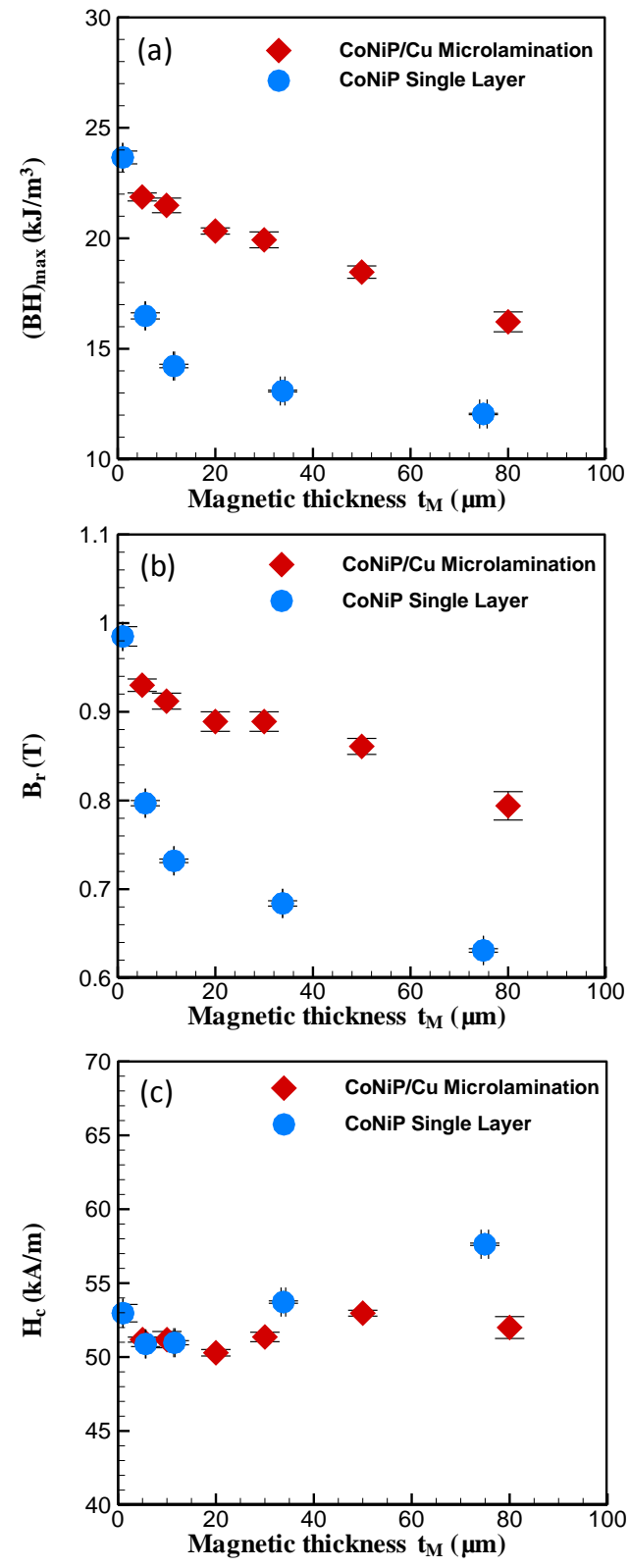

Figure 5: Comparison of the variation of in-plane magnetic properties: (a) maximum energy product, (b) remanence and (c) coercivity as a function of total magnetic film thickness $\left(t_{M}\right)$ between $\mathrm{CoNiP}(1 \mu \mathrm{m}) / \mathrm{Cu}(1 \mu \mathrm{m})$ microlamination and CoNiP single layer films.

\section{CONCLUSIONS}

A large-volume, high-energy-product laminated hard micromagnet enabled by a fully-integrated, CMOS-compatible fabrication approach has been successfully demonstrated utilizing the technique of sequential multilayer electroplating. The resultant laminated micromagnets showed a 30\% maximum energy density improvement at a large magnetic thickness of $80 \mu \mathrm{m}$ over nonlaminated counterparts. Further, by comparison with the state of the art of the same kind, the proposed micromagnet with laminated structure possessed superior areal magnetic energy density with which the capability of magnetic MEMS devices such as actuators and energy harvesters could be further improved. 

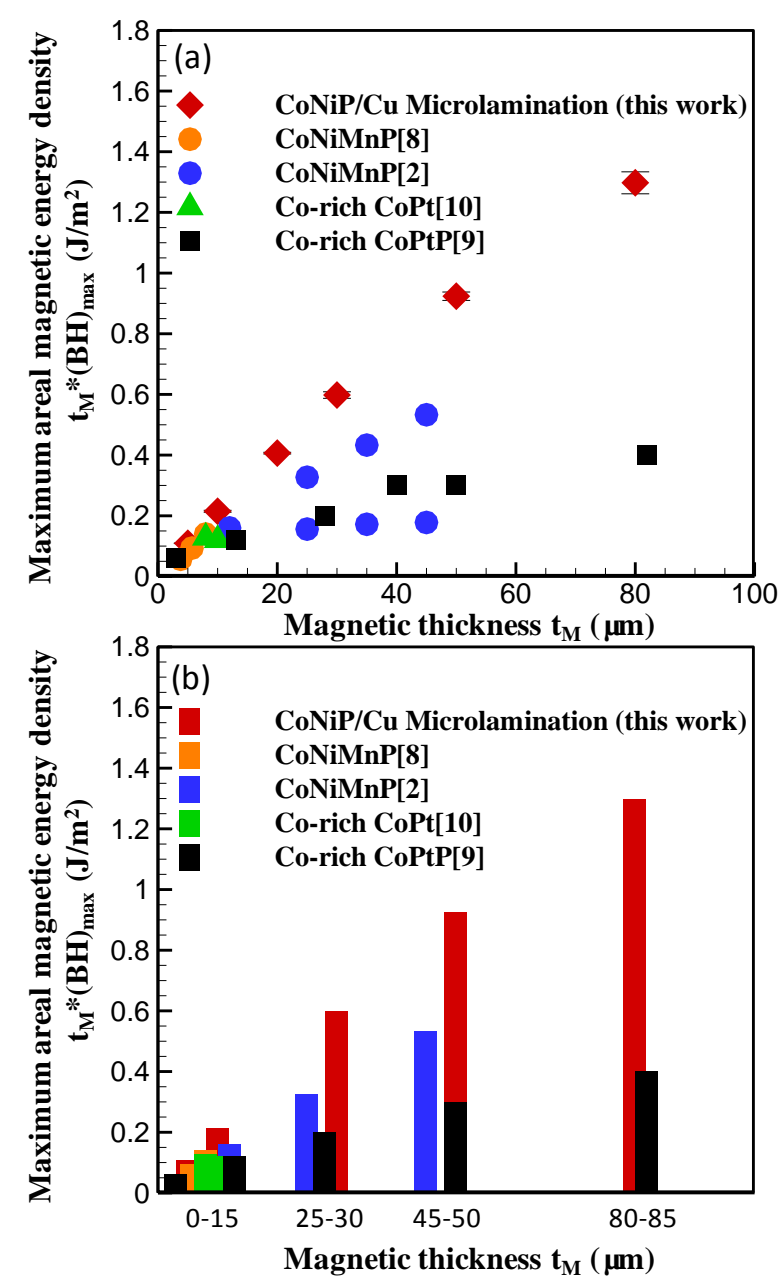

Figure 6: Comparison of film maximum areal magnetic energy density as a function of total magnetic film thickness $\left(t_{M}\right)$ between this work and data reported in literature: (a) full data and (b) data groupings for selected thicknesses.

\section{ACKNOWLEDGEMENTS}

This work was supported in part by the DARPA AXiS program (Grant \#N66001-11-1-4198). Travel support to the Hilton Head 2014 workshop has been generously provided by the Transducer Research Foundation. The authors would like to thank Dr. Jungkwun Kim for valuable discussions and Mr. Richard Shafer for helpful technical support on the electroplating robot.

\section{REFERENCES}

[1] D. Ettelt, P. Rey, M. Savoye, C. Coutier, M. Cartier, O. Redon, M. Audoin, A. Walther, P. Robert, and Y. Zhang, "A new low consumption 3D compass using integrated magnets and piezoresistive nano-gauges," in IEEE TRANSDUCERS'2011, pp. $40-43$.

[2] T. Liakopoulos, W. Zhang, and C. H. Ahn, "Electroplated thick CoNiMnP permanent magnet arrays for micromachined magnetic device applications," in IEEE MEMS'96, Proceedings, pp. 79-84.

[3] M. Han, Q. Yuan, X. Sun, and H. Zhang, "Design and fabrication of integrated magnetic MEMS energy harvester for low frequency applications," Microelectromechanical Systems, Journal of vol. 23, pp. 204 - 212, 2013.
[4] P. Wang, K. Tanaka, S. Sugiyama, X. Dai, X. Zhao, and J. Liu, "A micro electromagnetic low level vibration energy harvester based on MEMS technology," Microsystem technologies, vol. 15, pp. 941-951, 2009.

[5] D. P. Arnold and N. Wang, "Permanent magnets for MEMS," Microelectromechanical Systems, Journal of, vol. 18, pp. 1255-1266, 2009.

[6] G. Pattanaik, D. M. Kirkwood, X. Xu, and G. Zangari, "Electrodeposition of hard magnetic films and microstructures," Electrochimica acta, vol. 52, pp. 2755-2764, 2007.

[7] S. Piramanayagam, M. Matsumoto, and A. Morisako, "Thickness dependence of magnetic properties of $\mathrm{NdFeB}$ thin films with perpendicular magnetic anisotropy," Journal of magnetism and magnetic materials, vol. 212, pp. 12-16, 2000.

[8] X.-M. Sun, Q. Yuan, D.-M. Fang, and H.-X. Zhang, "Electrodeposition and characterization of CoNiMnP-based permanent magnetic film for MEMS applications," in IEEE NEMS' 2011, pp. 367-371.

[9] S. Kulkarni and S. Roy, "Deposition of thick Co-rich CoPtP films with high energy product for magnetic microelectromechanical applications," Journal of magnetism and magnetic materials, vol. 322, pp. 1592-1596, 2010.

[10] N. Wang and D. P. Arnold, "Thick electroplated Co-rich Co-Pt micromagnet arrays for magnetic MEMS," Magnetics, IEEE Transactions on, vol. 44, pp. 3969-3972, 2008.

[11] D. P. Arnold, "Review of microscale magnetic power generation," Magnetics, IEEE Transactions on, vol. 43, pp. 3940-3951, 2007.

[12] F. Herrault, W. Galle, R. Shafer, and M. Allen, "Electroplatingbased approaches for volumetric nanomanufacturing," Tech. Dig. Technologies for Future Micro-Nano Manufacturing, pp. 1-4, 2011.

[13] B. Peterson, W. Patterson, F. Herrault, D. Arnold, and M. Allen, "Laser-micromachined permanent magnet arrays with spatially alternating magnetic field distribution," Proceedings of PowerMEMS 2012, Atlanta, USA, pp. 319-322, 2012.

[14] D.-Y. Park, N. Myung, M. Schwartz, and K. Nobe, "Nanostructured magnetic CoNiP electrodeposits: structure-property relationships," Electrochimica acta, vol. 47, pp. 2893-2900, 2002.

[15] D. M. Kirkwood, G. Pattanaik, and G. Zangari, "Electrodeposited CoNiP films with perpendicular magnetic anisotropy," Journal of The Electrochemical Society, vol. 154, pp. D363-D368, 2007.

[16] J. Kim, M. Kim, F. Herrault, R. Shafer, J. Park, and M. Allen, "Nanolaminated permalloy core for high-flux, high-frequency ultracompact power conversion," Power Electronics, IEEE Transactions on, vol. 28, pp. 4376 - 4383, 2013.

[17] Q. Yao, W. Liu, W. B. Cui, F. Yang, X. G. Zhao, and Z. D. Zhang, "Growth mechanism and magnetic properties for the out-of-plane-oriented Nd-Fe-B films," Journal of Materials Research, vol. 24, pp. 2802-2812, 2009.

\section{CONTACT}

*Yuan Li, tel: +1-404-644-7238; yuanli1@ seas.upenn.edu 